\title{
RESEARCH
}

Open Access

\section{Metabolic syndrome increases senescence- associated micro-RNAs in extracellular vesicles derived from swine and human mesenchymal stem/stromal cells}

Yongxin Li ${ }^{1,2}$, Yu Meng ${ }^{3}$, Xiangyang Zhu' ${ }^{1}$ Ishran M. Saadiq ${ }^{1}$, Kyra L. Jordan ${ }^{1}$, Alfonso Eirin ${ }^{1}$ and Lilach O. Lerman ${ }^{1 *}$ (D)

\begin{abstract}
Background: The metabolic syndrome (MetS) is a combination of cardiovascular risk-factors, including obesity, hypertension, hyperglycemia, and insulin resistance. MetS may induce senescence in mesenchymal stem/stromal cells (MSC) and impact their micro-RNA (miRNA) content. We hypothesized that MetS also alters senescenceassociated (SA) miRNAs in MSC-derived extracellular vesicles (EVs), and interferes with their function.

Methods: EVs were collected from abdominal adipose tissue-derived MSCs from pigs with diet-induced MetS or Lean controls ( $n=6$ each), and from patients with MetS $(n=4)$ or age-matched Lean controls $(n=5)$. MiRNA sequencing was performed to identify dysregulated miRNAs in these EVs, and gene ontology to analyze their SA-genes targeted by dysregulated miRNAs. To test for EV function, MetS and Lean pig-EVs were co-incubated with renal tubular cells in-vitro or injected into pigs with renovascular disease (RVD, $n=6$ each) in-vivo. SA-b-Galactosidase and trichrome staining evaluated cellular senescence and fibrosis, respectively.

Results: Both humans and pigs with MetS showed obesity, hypertension, and hyperglycemia/insulin resistance. In MetS pigs, several upregulated and downregulated miRNAs targeted 5768 genes in MSC-EVs, 68 of which were SA. In MetS patients, downregulated and upregulated miRNAs targeted 131 SA-genes, 57 of which overlapped with pig-EVs miRNA targets. In-vitro, MetS-MSC-EVs induced greater senescence in renal tubular cells than Lean-MSC-EVs. In-Vivo, Lean-MSC-EVs attenuated renal senescence, fibrosis, and dysfunction more effectively than MetS-MSC-EVs.

Conclusions: MetS upregulates SA-miRNAs in swine MSC-EVs, which is conserved in human subjects, and attenuates their ability to blunt cellular senescence and repair injured target organs. These alterations need to be considered when designing therapeutic regenerative approaches.
\end{abstract}

Keywords: Metabolic syndrome, MSC, EV, RNA-sequencing, Senescence

* Correspondence: Lerman.Lilach@Mayo.edu

'Division of Nephrology and Hypertension, Mayo Clinic, 200 First Street SW, Rochester, MN 55905, USA

Full list of author information is available at the end of the article

(c) The Author(s). 2020 Open Access This article is licensed under a Creative Commons Attribution 4.0 International License, which permits use, sharing, adaptation, distribution and reproduction in any medium or format, as long as you give appropriate credit to the original author(s) and the source, provide a link to the Creative Commons licence, and indicate if changes were made. The images or other third party material in this article are included in the article's Creative Commons licence, unless indicated otherwise in a credit line to the material. If material is not included in the article's Creative Commons licence and your intended use is not permitted by statutory regulation or exceeds the permitted use, you will need to obtain permission directly from the copyright holder. To view a copy of this licence, visit http://creativecommons.org/licenses/by/4.0/. The Creative Commons Public Domain Dedication waiver (http://creativecommons.org/publicdomain/zero/1.0/) applies to the data made available in this article, unless otherwise stated in a credit line to the data. 


\section{Background}

Mesenchymal stem cells (MSCs) are multipotent cells with high proliferative, self-renewal, multi-lineage differentiation, and regenerative potential [1]. Emerging evidence suggests that MSCs isolated from fat and other tissue sources feature potent immunomodulatory and proangiogenic properties [2, 3], and thus serve important reparative functions.

Cellular senescence is an important cell fate, which entails apoptosis-resistance, stable replicative arrest, acquisition of a pro-inflammatory, tissue-destructive senescence-associated (SA) secretory phenotype (SASP), and shifted metabolism [4]. During this process, fatty acid oxidation is decreased, but protein synthesis and generation of reactive oxygen species increase [5]. SASP is linked to a variety of chronic diseases, geriatric syndromes, and metabolic dysregulation, including metabolic syndrome (MetS) and atherosclerosis.

Characterized by dyslipidemia, obesity, insulin resistance, inflammation, as well as hypertension, MetS affects a large number of people worldwide [6]. The presence of MetS has been associated with atherosclerosis renovascular disease (RVD) and chronic kidney disease [7]. Accumulating evidence has shown that the proliferation capacity of MSCs is inversely correlated with cellular senescence and apoptosis in MetS patients [8]. We have also previously shown that MetS impairs the functionality of MSCs, increases their cellular senescence [9], and alters their genetic and protein content [10-12].

Previous studies [13, 14] showed that MSCs bestow their reparative effects by releasing extracellular vesicles (EVs), including micro-vesicles and exosomes. EVs can alter transcription profiles in recipient cells, and modulate tissue metabolism and cellular pathways. Our group recently demonstrated that EVs released by pig MSCs are selectively packed with micro-RNAS (miRNAs), mRNAs, and proteins, which have the capacity to modify selective pathways in recipient cells $[10,12,15,16]$. miRNAs are small non-coding RNAs that regulate gene expression post-transcriptionally. We have shown that MetS modulates MSC expression of senescenceassociated (SA)-miRNAs [12], but whether this alteration extends to their paracrine EVs, or interferes with their ability to suppress SA mechanisms in target cells, remains known.

The current study was therefore designed to test the hypothesis that MetS upregulates SA-miRNAs in adipose tissue MSCs-derived EVs in pigs, and impairs their repair capacity. Furthermore, we hypothesized that this adverse dysregulation would also be conserved in MSCs obtained from human subjects with MetS. For this purpose, we comprehensively evaluated the MSC-derived EV miRNA expression in both human subjects and pigs with MetS using high-throughput RNA sequencing, and injected pig EVs into injured kidneys of a pig model with RVD. A chief finding of our study is that miRNA spectrum in MSC-derived EVs in MetS might be involved in senescence-regulation. Importantly, we found that MetS stifles the ability of MSC-derived EVs to ameliorate senescence and fibrosis in RVD pig kidneys, and increases their propensity to induce senescence in target cells in vitro. These observations may have important implications for the reparative potency of endogenous EVs and for the utility of autologous exogenous EVs in subjects with MetS.

\section{Materials and methods}

Animals and protocol

Animal studies were approved by the Institutional Animal Care and Use Committee. For EV characterization, 12 three-months-old female domestic pigs (Manthei Hog Farm, Elk River, MN) were observed for 16 weeks in two groups. Control pigs (Lean) were fed a standard chow (13\% protein, $2 \%$ fat, $6 \%$ fiber, Purina Animal Nutrition LLC, MN), and MetS pigs a metabolicatherosclerotic diet (5B4L, protein 16.1\%, ether extract fat $43.0 \%$, and carbohydrates $40.8 \%$, Purina Test Diet, Richmond, IN) ( $n=6$ each) [17]. After completion of diet, systemic parameters including body weight, blood pressure (measured with an intra-arterial catheter), and venous levels of cholesterol, triglycerides, fasting glucose, and insulin were measured by standard procedures [18]. MSCs and EVs were then collected.

\section{Patient population}

Healthy subjects and MetS patients ( $n=5$ in each group) were recruited in the First Hospital Affiliated to Jinan University (Guangdong, China). The study followed the Declaration of Helsinki. Both the project and molecular testing were approved by the Institutional Research Ethics Committee, and written informed consent obtained from all subjects. All study participants were reviewed for medical history.

Inclusion criteria for MetS patients included age $>18$ years old and MetS diagnosis, based on the criteria of the International Diabetes Federation. The diagnosis of the MetS was based on obesity (excessive waist circumference or BMI $>30 \mathrm{~kg} / \mathrm{m} 2$ ) and two or more of the following factors: abnormal lipids metabolism (HDL cholesterol $<40 \mathrm{mg} / \mathrm{dL}$ in males and $<50 \mathrm{mg} / \mathrm{dL}$ in $\mathrm{fe}$ males, triglycerides $\geq 150 \mathrm{mg} / \mathrm{dL}$ ), systolic blood pressure $\geq 130 \mathrm{mmHg}$ or diastolic blood pressure $\geq 85$ mmHg; fasting plasma glucose concentration $\geq 100 \mathrm{mg} /$ $\mathrm{dl}$ or previously diagnosed hypertension or type- 2 diabetes. Exclusion criteria for MetS patients included drug abuse, heavy smoking, cancer, severe heart valves diseases, and any kind of severe systemic diseases. 
Inclusion criteria for healthy controls included age > 18 years, overall healthy individuals, who had abdominal fat harvested for cosmetic reasons. Exclusion criteria for healthy controls included drug abuse and heavy smoking.

Blood samples were collected prior to surgery for assessment of metabolic and renal function in the clinical laboratories of the First Hospital Affiliated to Jinan University. Estimated glomerular filtration rate (eGFR) was calculated by the MDRD eGFR Equation [19].

\section{MSCs and EV harvesting}

Abdominal subcutaneous adipose tissue collection, MSC isolation and culture, and EV collection were performed as previously described [17].

Pigs were euthanized with a lethal intravenous dose of $100 \mathrm{mg} / \mathrm{kg}$ of sodium pentobarbital (Fatal Plus, Vortech Pharmaceuticals, Dearborn, MI, USA) and subcutaneous abdominal adipose (5-10 g) tissue immediately collected. Fat was digested in collagenase-H, filtered with $0.2-\mathrm{mm}$ syringe filter, and cultured in advanced minimal essential medium (GIBCO/Invitrogen, Grand Island, NY, USA) supplemented with $5 \%$ platelet lysate for about 3 weeks. EVs were isolated from supernatants of passage 3 MSCs (at least $10^{7}$ cells) by ultracentrifugation, as previously described $[10,11]$. Then the samples were centrifuged (2000 $\mathrm{g}$ for $20 \mathrm{~min}$ ), and the supernatant collected and subsequently centrifuged $(100,000 \mathrm{~g}$ for $1 \mathrm{~h})$ at $4{ }^{\circ} \mathrm{C}$. EVs were collected, suspended (wash-buffer medium 199) and centrifuged again $(100,000 \mathrm{~g}$ for $1 \mathrm{~h})$.

MSCs were previously characterized by the expression of CD44, CD90, and CD105, and lack of expression of CD45, CD34, CD14, using immunofluorescent staining and flow-cytometry [17]. Isolated EVs were then characterized based on the expression of EV (CD9, CD29, CD63) and MSC (CD73, CD105) markers by Western blot [17]. In human subjects, adipose tissue (5-10 g) was collected during bariatric (MetS patients) or cosmetic (Lean) surgeries. The samples were placed on ice and processed for MSC harvesting, expansion, and EV collection using identical methods to those employed in pig studies.

\section{MiRNA sequencing and data analysis}

Human EV total RNA libraries were prepared with QIAseq Stranded Total RNA Kit. MiRNA sequencing libraries prepared with QIAseq miRNA Library Kit were sequenced using an Illumina NGS system (MiSeq Personal Sequencer, NextSequence500, HiSeq 1000, HiSeq 1500, HiSeq 2000, HiSeq 2500, and GaIIx). The data were analyzed with CLC (Biomedical) Genomics Workbench.

Sequencing RNA libraries of pig EVs were prepared according to the manufacturer's protocol (TruSeq RNA Sample Prep Kit v2, Illumina). The pig EV data were analyzed using the CAP-miRSeq-v1.1 workflow [20]. The workflow starts with unaligned FASTQs, which generates aligned BAMs, and then excel sheets containing both raw and normalized known mature miRNA expression counts. The R-based tool from Bioconductor, edgeR2.6.2 was used to perform the differential expression analysis to identify miRNAs enriched in MetS-EVs compared to Lean-EVs (fold-change $>2.0$ and $p<0.05$ ). ComiR and TargetScan 7.1 were used to predict target genes of significantly upregulated and downregulated miRNAs. Subsequent functional annotation-clustering analysis utilized the DAVID 6.7 database.

\section{Validation of miRNA expression}

To validate expression of representative miRNAs in pig EVs, the expression of miR-199a-5p, miR-132 and miR99b was also measured by quantitative polymerase chain reaction (qPCR). Total RNA was isolated from MSCderived EV samples [21], and probed with primers (TermoFisher Scientifc, Minneapolis, MN, USA; Catalog Numbers: miR-99b: 002196, miR-132: 000457, miR199a-5p:000498).

\section{In vitro EV efficacy}

For in vitro studies, pig renal tubular cells (PK1 cells, ATCC, Manassas) were cultured in Medium-199 (Gibco BRL, USA) containing 3\% FBS15. EVs were incubated in $2 \times 10^{-6} \mathrm{M}$ PKH26 for $5 \mathrm{~min}$ and culture media with $10 \%$ EV-depleted FBS $(2 \mathrm{ml})$ added to stop the action. Then EVs were centrifuged $(100,000 \mathrm{~g}$ for $1 \mathrm{~h})$ and resuspended in DPBS $(5 \mathrm{ml})$. Pre-labeled EV-rich fractions $\left(75 \times 10^{\wedge} 12 / \mathrm{mL}\right)$ were incubated with PK1 cells for $48 \mathrm{~h}$, and tracked to confirm engraftment. Staining for SASpider-ß-Gal in PK1 cells was performed as previously described [22] following manufacturer's protocol (Cat\#SG04-01, Dojindo).

\section{In vivo $\mathrm{EV}$ efficacy}

The pig RVD model and EV injection was performed as previously described $[17,23]$. Twenty-four additional female domestic pigs were randomized into four groups: Lean, RVD, RVD + Lean-MSC-EVs and RVD + MetSMSC-EVs ( $n=6$ each). Lean pigs were fed a standard chow, and RVD pigs a metabolic-atherosclerotic diet. Six weeks after initiation of diet, unilateral renal artery stenosis was induced in 18 RVD pigs by placing an irritant coil in the main renal artery [23] to achieve gradual arterial narrowing.

Six weeks after induction of RVD, EV-rich fractions from the Lean and MetS MSCs were labeled with the red fluorescence dye PKH26 (Sigma-Aldich, ST. Louis, $\mathrm{MO})$. After incubating in $2 \times 10^{-6} \mathrm{MPKH} 26$ for $5 \mathrm{~min}$, EVs were added with culture media with $10 \% \mathrm{EV}$ depleted FBS $(2 \mathrm{ml})$ to stop the action according to instructions. EVs were centrifuged $(100,000 \mathrm{~g}$ for $1 \mathrm{~h})$ and 
re-suspended in DPBS ( $5 \mathrm{ml})$. In two RVD groups, Lean or MetS EVs $\left(100 \mu \mathrm{g}\right.$ or $\left.10^{11}\right)$ in $10 \mathrm{ml}$ were then injected (each in 6 RVD pigs) through a catheter placed in the stenotic renal artery under fluoroscopic guidance. The other groups underwent renal angiography to determine the degree of stenosis, and all pigs were allowed to recover.

Four weeks after EV injection, blood pressure was measured using an intra-arterial catheter, the pigs were euthanized, and the kidneys harvested. The labeled EVs were tracked in frozen sections of RVD kidneys to confirm engraftment [11]. Staining for SA-Spider-B-Gal was performed following manufacturer's protocol (Cat\#SG04-01, Dojindo), on $10 \mu \mathrm{m}$ frozen sections counter-stained with eosin. Senescence (green) area in kidney was measured by using the Image-J software in 10 individual fields, calculated as percent of kidney area, and averaged for all fields.

Fibrosis was assessed using Masson's trichrome staining with a modified IMEB stain kit (K7298, IMEB, SanMarcos, CA). Images were visualized with a Zeiss microscope. Fibrosis area (blue) in kidney was measured by MATLAB in 10 individual fields and averaged.

\section{Statistical analysis}

Statistical analysis was performed using JMP 14.0 (SAS Institute, Cary, NC). Data were expressed as mean \pm standard deviation. Comparisons among groups were performed using unpaired Student's t-test and ANOVA. Nonparametric tests (Wilcoxon and Kruskal Wallis) was used when data did not follow a Gaussian distribution. Statistical significance was accepted if $p \leq 0.05$.

\section{Results}

\section{Systemic characteristics of Lean and Mets pigs}

Table 1 shows the systemic characteristics of the Lean and MetS pigs. Compared with Lean, after 16 weeks of diet MetS pigs showed higher body weight, blood pressure, and cholesterol levels, including total cholesterol,

Table 1 Systemic characteristics in experimental pig groups $(n=6$ each) at 16 weeks

\begin{tabular}{lll}
\hline Parameter & Lean & MetS \\
\hline Body weight $(\mathrm{Kg})$ & $71.4 \pm 10.8$ & $90.8 \pm 1.8^{*}$ \\
Mean blood pressure $(\mathrm{mmHg})$ & $95.1 \pm 9.9$ & $119.8 \pm 3.9^{*}$ \\
Total cholesterol $(\mathrm{mg} / \mathrm{dl})$ & $80.8 \pm 6.4$ & $398.1 \pm 60.0^{*}$ \\
LDL cholesterol $(\mathrm{mg} / \mathrm{dl})$ & $33.1 \pm 5.2$ & $378.1 \pm 137.6^{*}$ \\
Triglycerides $(\mathrm{mg} / \mathrm{dl})$ & $7.7 \pm 1.2$ & $14.6 \pm 1.5^{*}$ \\
Fasting glucose $(\mathrm{mg} / \mathrm{dl})$ & $127.1 \pm 14.2$ & $118.8 \pm 18.0$ \\
Fasting insulin $(\mu \mathrm{U} / \mathrm{ml})$ & $0.4 \pm 0.1$ & $0.7 \pm 0.1^{*}$ \\
HOMA-IR score & $0.7 \pm 0.1$ & $1.7 \pm 0.3^{*}$ \\
\hline
\end{tabular}

MetS metabolic syndrome, LDL Low-density lipoprotein, HOMA-IR Homeostasis model-assessment of insulin resistance

${ }^{*} p \leq 0.05$ vs. Lean
LDL, and triglycerides. Furthermore, HOMA-IR score and fasting insulin were higher in MetS vs. Lean, although fasting glucose levels were unchanged. These findings indicate development of MetS.

\section{MSCs-derived EV miRNAs and targeted SA gene regulation in pig models}

We then analyzed miRNAs in MetS pig MSC-derived EVs. Four miRNAs (miR-132, miR-199a-5p, miR-212, miR-374a-3p) were upregulated and four (miR-99b, miR-378, miR-504, miR-186) downregulated in MetSMSC-derived EVs compared with Lean MSCs-derived EVs (Fig. 1a). Altogether, these dysregulated miRNAs target 5700 genes, of which 68 are related to cellular senescence. Functional annotation clustering and pathway analysis of these 68 genes, including MAPK1, PTEN, and MTOR, confirmed their relationship with cellular senescence, cell cycle, regulation of metabolic process, and MAPK signaling pathways (Fig. 1b). Expression patterns of miR-199a-5p, miR-132 and miR-99b were subsequently confirmed by PCR (Fig. 1c). The SAgenes targeted by dysregulated miRNAs are detailed in Supplementary Material (Table 1s).

\section{Systemic characteristics of Lean and Mets patients}

Table 2 shows the demographic, clinical, and laboratory characteristics of the study patients. Body mass index, systolic, and diastolic blood pressures were all significantly higher in the MetS than Lean group, whereas age and sex were similar. Total cholesterol, low-density lipoprotein, insulin, and hemoglobin $\mathrm{A} 1 \mathrm{C}$ levels were also higher in MetS compared to Lean, underscoring development of MetS. Elevated eGFR was consistent with development of hyperfiltration that characterizes obese individuals.

\section{MSCs-derived EVs miRNAs and targeted SA gene regulation in MetS patients}

We further analyzed miRNAs dysregulated in human MetS MSC-derived EVs. Four miRNAs (miR-136-3p, miR-4798-5p, miR-12,136, miR-222-3p) were downregulated and nine (miR-630, miR-144-3p, miR-143-5p, miR4787-3p, miR-769-5p, miR-8074, miR-181a-5p) upregulated in MetS- compared with Lean MSC-derived EVs (Fig. 2a). Altogether, these dysregulated miRNAs target 5700 genes, of which 131 are related to cellular senescence. Functional annotation clustering and pathway analysis of these 131 genes, including PTEN, FOXO3, and MAPK1, showed their relationship with cellular senescence, cell cycle, metabolic processes and apoptosis pathway (Fig. 2b). SA-genes targeted by dysregulated miRNAs in human subjects with MetS are listed in Supplementary Material (Table 2s). 
(A)

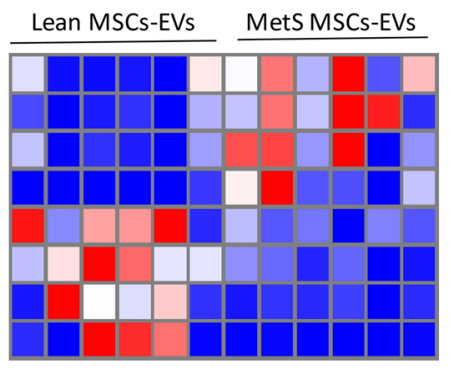

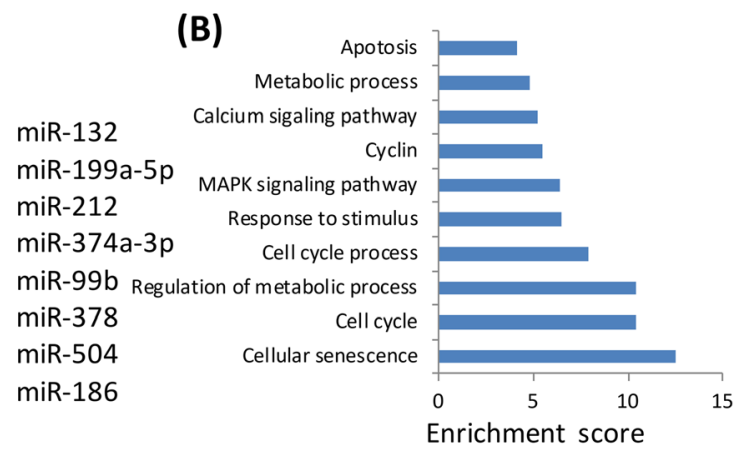

(C)

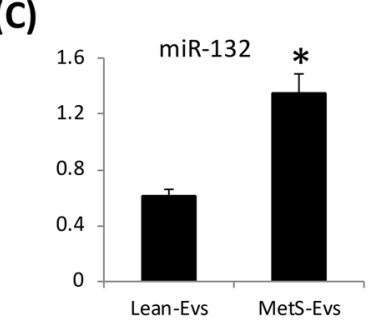

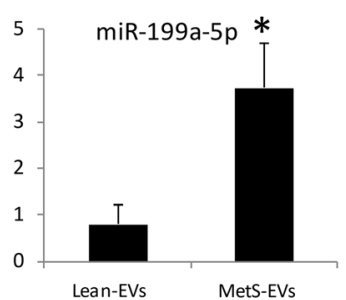

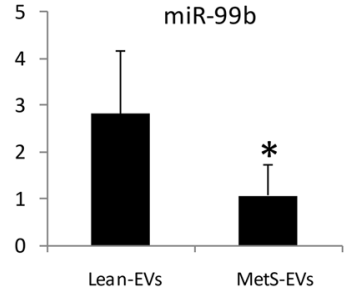

Fig. 1 MicroRNA (miRNA) profile in Lean and MetS MSC-EVs in pigs. Heat map showing four upregulated (top) and four downregulated (bottom) miRNAs in MetS compared with Lean MSC-EVs in pigs. Enrichment of functional pathway of the 68 senescence genes targeted by dysregulated pig miRNAs detected using DAVID 6.7. Expression of miR-132, miR-199a-5p, and miR-99b assessed by qPCR was concordant with miRNA-seq findings in pigs

Further comparison of targeted SA-genes of differentially expressed miRNAs in human and swine MetSMSCs identified 57 common genes, accounting for about $40 \%$ of the targeted human SA-genes and $84 \%$ of the pig SA-genes (Fig. 2c). Functional annotation clustering and pathway analysis of these 57 common genes disclosed their association with cellular senescence, regulation of metabolic processes, cell cycle, responses to stimulus,

Table 2 Clinical, laboratory, and demographic data of Lean and Mets patients

\begin{tabular}{lll}
\hline parameter & Lean & Mets \\
\hline Number & 5 & 4 \\
Age (years) & $24.2(21-29)$ & $29.3(24-32)$ \\
Gender (female/male) & $3 / 2$ & $2 / 2$ \\
Body mass index & $19.1 \pm 0.8$ & $60.7 \pm 16.2^{*}$ \\
SBP (mmHg) & $110.6 \pm 11.7$ & $147 \pm 12.5^{*}$ \\
DBP (mmHg) & $63.4 \pm 5.1$ & $93.8 \pm 6.3^{*}$ \\
Hemoglobin A1C(\%) & $5.3 \pm 0.2$ & $6.9 \pm 1.3^{*}$ \\
Total cholesterol (mmol/l) & $4.4 \pm 0.4$ & $5.3 \pm 0.9^{*}$ \\
Low-density lipoprotein (mmol/l) & $1.6 \pm 0.3$ & $3.0 \pm 0.4^{*}$ \\
Blood urea nitrogen (mmol/l) & $3.7 \pm 0.4$ & $5.5 \pm 1.6^{*}$ \\
GFR (ml/min/1.73m ${ }^{2}$ ) & $112.8 \pm 34.4$ & $214.5 \pm 40.4^{*}$ \\
Cystatin-C (mg/1) & $0.9 \pm 0.1$ & $1.4 \pm 0.2^{*}$ \\
\hline
\end{tabular}

SBP systolic blood pressure, DBP diastolic blood pressure, GFR estimated glomerular filtration rate

${ }^{*} P<0.05$ vs Lean apoptosis, and MAPK signaling pathways (Fig. 2d), which are clustered with SA-genes. The common dysregulated miRNAs targeted SA genes are listed in Supplementary Material (Table 3s).

\section{Systemic characteristics of Lean and RVD pigs}

The systemic characteristic of Lean and RVD pigs are summarized in Table 3. Treated or untreated RVD pigs developed a similar degree of significant renal artery stenosis and elevated blood pressure. Furthermore, in untreated RVD pigs serum creatinine was significantly elevated compared to Lean pigs.

\section{MetS attenuates the repair capacity of MSC-derived EVs in vitro and in vivo}

Labeled EV-rich fractions (red) were identified in PK1 cells (Fig. 3b), confirming their uptake. Senescent cells are characterized by increased enzymatic activities of the lysosomal hydrolase SA-ß-gal. SA-ß-gal staining remained similar to control in PK1 cells co-cultured with Lean-MSC-EVs, but increased significantly in PK1 cells co-cultured with MetS-MSC-EVs (Fig. 3a), indicating that they induced senescence.

RVD kidneys showed elevated SA- $ß$-gal and trichrome staining (Fig. 3c), indicating development of cellular senescence and fibrosis, as we have shown [7]. In stenotic pig kidneys injected with Lean-MSC-EVs or MetS-MSCEVs, uptake of labeled EVs (red) was identified (Fig. 3d). 


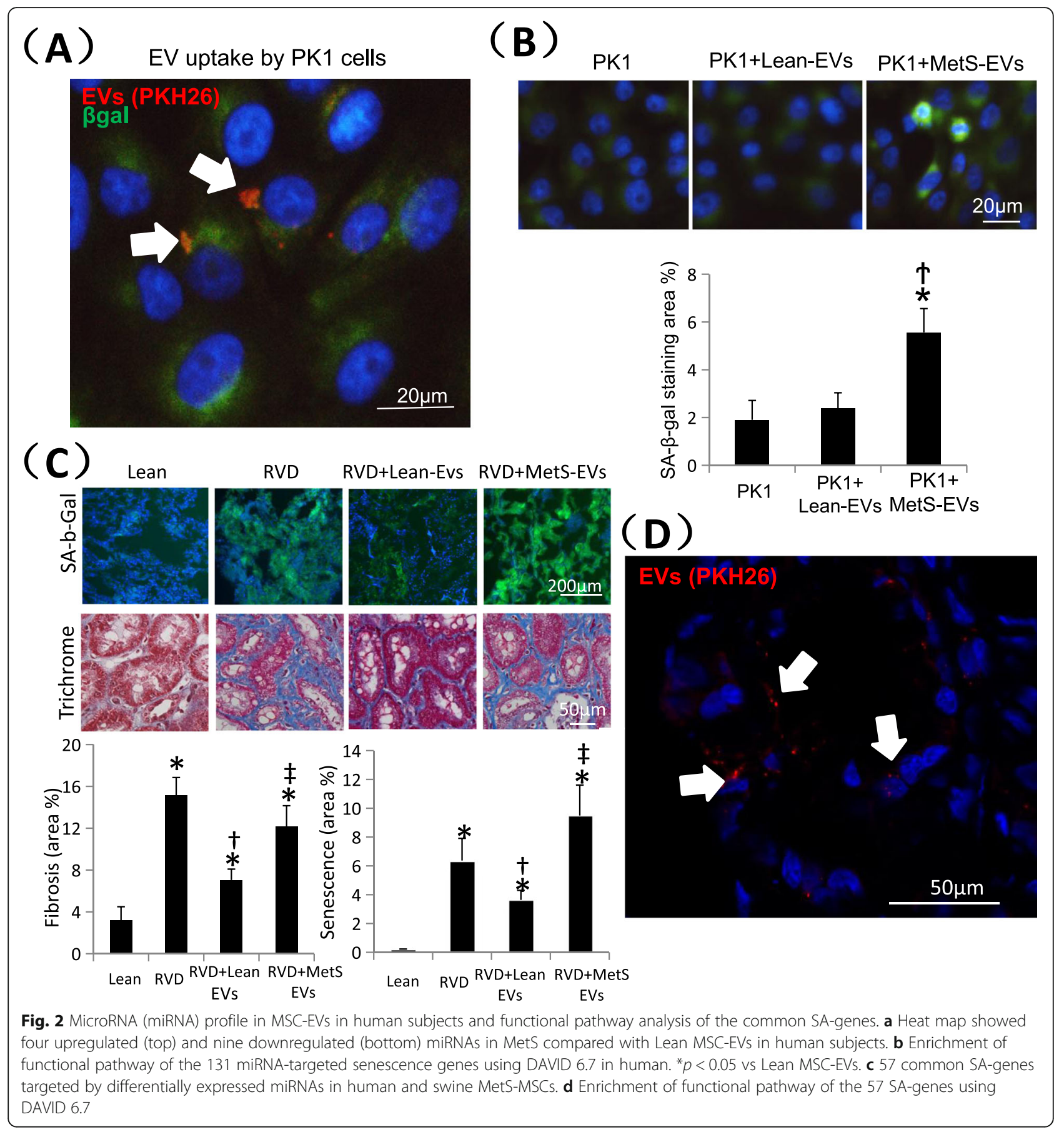

Compared with RVD, delivery of Lean-EVs significantly blunted, although not normalized, cellular senescence and fibrosis. On the other hand, delivery of MetS-EVs did not decrease either cellular senescence or fibrosis, which remained not different from the untreated RVD group, and elevated compared with the RVD + Lean-EVs group. Furthermore, Lean-EVs, but not MetS-EVs, also significantly decreased serum creatinine levels (Table 3).

\section{Discussion}

The current study shows that MetS increases the content of senescence-associated miRNAs in MSCs-derived EVs in both a pig model and human subjects. While the specific SA-miRNAs differed between humans and pigs with MetS, their MSCs-derived EVs shared 57 common SA target genes. These observations suggest that increased content of SA-miRNAs in MSCs-derived EVs in MetS is conserved in pigs and humans. Furthermore, we 
Table 3 Systemic characteristics and single-kidney function in pigs with renovascular disease (RVD) 4 weeks after treatment with MSC-derived extracellular vesicles (EVs)

\begin{tabular}{lllll}
\hline Parameter & Lean & RVD & RVD + Lean-EVs & RVD + MetS-EVs \\
\hline SBP $(\mathrm{mmHg})$ & $127.0 \pm 15.3$ & $159.8 \pm 22.3^{*}$ & $142.3 \pm 12.3^{*}$ & $170.8 \pm 12.6^{*}$ \\
DBP $(\mathrm{mmHg})$ & $92.8 \pm 11.0$ & $115.0 \pm 17.7^{*}$ & $108.0 \pm 10.5^{*}$ & $105.7 \pm 4.5^{*}$ \\
MAP $(\mathrm{mmHg})$ & $90.7 \pm 4.4$ & $131.9 \pm 17.3^{*}$ & $116.8 \pm 12.5^{*}$ & $127.4 \pm 6.3^{*}$ \\
Degree of stenosis $(\%)$ & 0 & $64.2 \pm 19.6^{*}$ & $65.0 \pm 8.4^{*}$ & $65.8 \pm 6.6^{*}$ \\
Serum creatinine $(\mathrm{mg} / \mathrm{dL})$ & $1.44 \pm 0.21$ & $1.77 \pm 0.23^{*}$ & $1.48 \pm 0.29 \dagger$ & $1.76 \pm 0.26^{*} \neq$ \\
\hline
\end{tabular}

$S B P$ systolic blood pressure, $D B P$ diastolic blood pressure, MAP mean arterial pressure

$n=6$ each group. ${ }^{*} p<0.05$ vs. Lean; $t p<0.05$ vs. RVD; $\neq p<0.05$ vs. RVD + Lean-EVs

found that pig MetS-EVs induce cellular senescence in PK1 cells, and show dampened potency for attenuation of cellular senescence and fibrosis in RVD pig kidneys. Therefore, the altered SA-content profile of MSCsderived EVs in MetS may interfere with their ability to blunt senescence in target cells.

MSC-derived EVs mediate the paracrine function of their parental MSCs by transferring physiologicallyrelevant components from MSCs to recipient cells. The current study extends our previously observation that MetS alters miRNA spectrum in MSCs that might participate in regulation of cellular senescence within the parent MSCs [24], and shows that MetS also modifies miRNA content in their daughter EV paracrine vectors, which might modulate cellular senescence within target cells that MSCs communicate with. We identified miRNAs dysregulated in MetS-MSCs-derived EVs compared with Lean MSC-derived EVs, which target 68 SAgenes. Further functional annotation clustering and pathway analysis showed the relationship of these 68 genes with cellular senescence, cell cycle, regulation of metabolic process, and MAPK signaling pathway, which

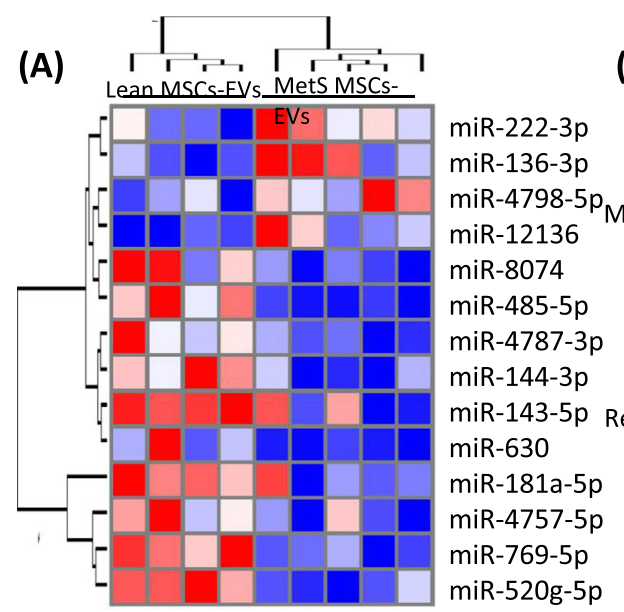

(C)

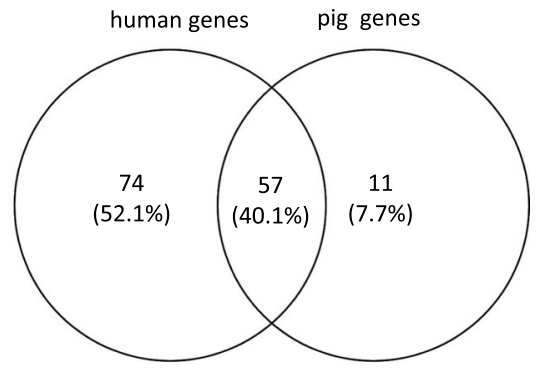

(B)

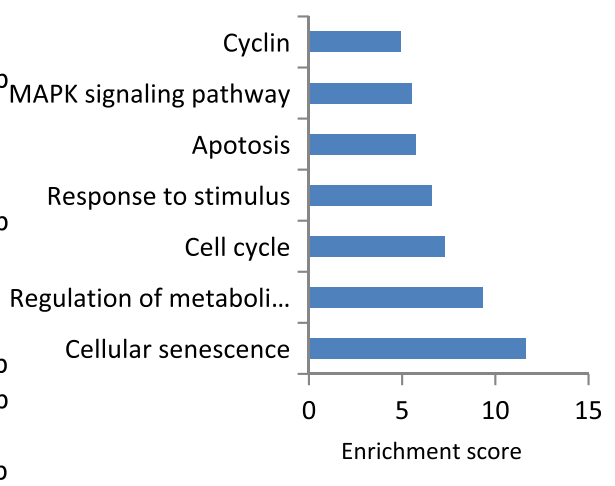

(D)

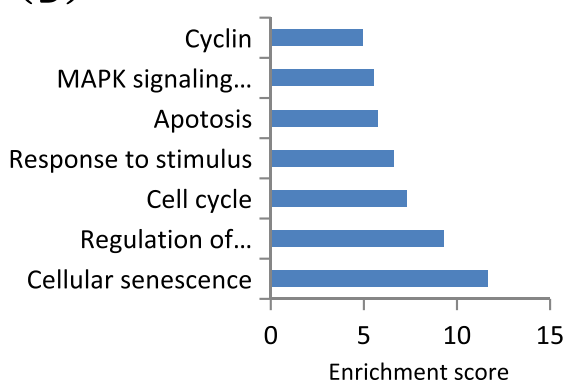

Fig. 3 Effects of MSC-derived EVs in PK1 cells and pig kidney. a Co-cultured with MetS MSC-EVs, PK1 cells showed higher senescence.* $p<0.05$ vs PK1,$+p<0.05$ vs PK1 + Lean-EVs. b PKH-26-labeled EVs (red) were detected in PK1 cells. c Representative kidney staining with immunofluorescent SA-b-Gal (left top) and trichrome (left bottom), and respective quantification. Lean EVs attenuated cellular senescence and fibrosis in vivo in injured kidneys, whereas MetS EVs failed to blunt them. $\mathbf{d}$ Pkh-26-labeled EVs (red) were detected in frozen section in the RVD kidney. ${ }^{*} p<0.05$ vs Lean, $\nmid p<0.05$ vs RVD, $\neq p<0.05$ vs RVD + Lean-EVs 
are also the major senescence pathways targeted by miRNAs in MetS-MSCs in pigs [12]. Hence, miRNAs in EVs might have a similar potential to their parent MSCs in regulating cellular senescence.

This study also underscores that notion that while miRNAs enriched in EVs may be diverse across species, they share a potential to regulate similar pathways in recipient cells, such as proliferation, inflammation, and angiogenesis $[25,26]$. While dysregulated miRNAs were not identical in human and pig adipose tissue-derived MSC-EVs, they had comparable targets involved in cellular senescence, cell cycle, metabolic processes, and apoptosis pathways. Remarkably, despite speciesdependent heterogeneity, our pig model and patients with MetS shared 57 SA-genes, accounting for $40 \%$ of the human targeted SA-genes and $84 \%$ of the pig genes, highlighting the similarity of MetS-induced alterations in both species.

A recent study showed that small EVs may contribute to paracrine senescence as key regulators of intercellular communication [27]. EVs play important roles in cell-tocell communication of their parent cells by transferring miRNAs, DNA, proteins, mRNAs, lipids, and organelles to recipient cells [28]. Among the dysregulated miRNAs, several have been reported to participate in senescence by specific pathways. For example, miR-222-3p can induce cell-cycle phase-arrest and telomere erosion, establishing a senescent phenotype by promoting accumulation of human dermal fibroblasts in G1/S cell cycle phase [29]. On the other hand, miR-143-5p activates AMPK signaling and promotes apoptosis and senescence by targeting the eEF2 gene [30]. Similarly, we found that MetS-EVs could promote cellular senescence in pig PK1 cells in vitro, which might be induced by the dysregulated miRNAs.

The ability of EVs to regulate renal cellular senescence in RVD was unknown. We observed that cellular senescence was increased in untreated RVD kidneys, as we have found before in a different pig model of dyslipidemic RVD [7] and in kidneys of obese mice [31]. The current study demonstrates that EVs derived from healthy (Lean) MSCs attenuate cellular senescence detected by SA-B-gal in injured post-stenotic swine kidneys. Equivalently, we have recently reported that MSCderived EVs attenuated cellular senescence and inflammation in the pig myocardium [17]. Finally, similar to cellular senescence, Lean-EVs, but not MetS-EVs, attenuated renal fibrosis. While this does not mandate causality, this observation is consistent with a recent study linking cellular senescence with development of renal fibrosis in tubular epithelial cells [32]. Importantly, decreased tissue injury by Lean-EVs translated into improved kidney function. Overall, our findings suggest that altered content of miRNA targeting SA-genes might be functionally meaningful and restrict their reparative effectiveness.

Our study combined and comprehensively analyzed the data from human and pig models, and has a number of strengths. Using next-generation sequencing analysis, we identified differential miRNA expression signatures in MSC-derived EVs in MetS compared with Lean human subjects and pigs, and subsequently demonstrated their decreased efficacy to attenuate cellular senescence in vivo and in vitro. Our functional analysis focused on dysregulated miRNAs and their targeted SA-genes, and succeeded to demonstrate the role of miRNAS packed in EVs in cellular senescence. The limitations of our study included a relatively small sample size, and short duration of MetS and RVD in pigs, although importantly our patients were also relatively young. In addition, the MSCs and EVs used in this study were harvested in strict adherence with our routine protocols [17], without undergoing further de novo characterization. We also used for RNA-Seq EVs from third passage of MSCs, because at late passages MSCs may become senescent [33]. Further studies are needed to explore in detail genes and molecules that regulate these pathways, as well as techniques to blunt them. The number of delivered EVs $\left(10^{11}\right)$ resembles the overall concentration of EVs in the systemic circulation $\left(10^{9}-10^{12}\right)$ [34], yet MSC-derived EV are especially endowed with reparative properties and were injected into the renal artery, and thereby achieved momentous effects.

\section{Conclusions}

MetS dysregulates in MSC-derived EVs miRNAs that may participate in regulating cellular senescence in both pigs and human subjects. These alterations result in promotion of senescence by MetS-EVs, and their incompetence to ameliorate senescence and fibrosis in injured kidneys. These studies suggest that miRNAs in MSCderived EVs may regulate cellular senescence and may have important implications for both the endogenous cellular repair system, as well as for the use of autologous EVs as a therapeutic regenerative strategy.

\section{Supplementary information}

Supplementary information accompanies this paper at https://doi.org/10. 1186/s12964-020-00624-8.

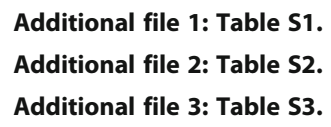

\section{Abbreviations}

MetS: Metabolic syndrome; MSC: Mesenchymal stem/stromal cells; SA: Senescence-associated; EVs: Extracellular vesicles; RVD: Renovascular disease; miRNA: micro-RNA; SASP: Senescence-associated secretory phenotype 


\section{Acknowledgments}

We would like to thank to Nephrology and Hypertension staff members for helpful discussions.

\section{Authors' contributions}

YL: experimental design, collection and assembly of data, data analysis and interpretation, manuscript writing. YM: collection and assembly of data, assembly of data, data analysis and interpretation. AE: conception and design, assisted with data collection and analysis, manuscript editing. XYZ: collection and assembly of data, assembly of data. IMS: collection and assembly of data, assembly of data. KLJ: collection and assembly of data, assembly of data. LOL: conception and design, financial support, manuscript editing. All authors read and approved the manuscript.

\section{Funding}

This study was partly supported by NIH grant numbers DK120292, DK122734, DK102325, AG062104, DK106427, and DK122137, the Natural Science Foundation of GuangDong 2018A030313527, and the Basic and Applied basic research Foundation of Guangdong Province 2019A1515010176.

\section{Availability of data and materials}

The authors declare that data supporting the findings of this study are available within the article and its Additional information files.

\section{Ethics approval and consent to participate}

All samples were obtained under a protocol approved by the First Hospital Affiliated to Jinan University Institutional Research Ethics Committee (Guangdong, China). All volunteers provided written informed consent.

\section{Competing interests}

Dr. Lerman receives grant funding from Novo Nordisk, and is an advisor to Weijian Technologies and AstraZeneca. The authors declared no potential conflicts of interest with respect to the research, authorship, and/or publication of this article.

\section{Author details}

'Division of Nephrology and Hypertension, Mayo Clinic, 200 First Street SW, Rochester, MN 55905, USA. ${ }^{2}$ Dapartment of Vascular Surgery, The Affiliated Hospital of Qingdao University, Qingdao 266000, People's Republic of China. ${ }^{3}$ Department of Nephrology, The First Hospital Affiliated to Jinan University, Guangzhou 510630, People's Republic of China.

Received: 17 April 2020 Accepted: 2 July 2020

\section{Published online: 12 August 2020}

\section{References}

1. Charbord P. Bone marrow mesenchymal stem cells: historical overview and concepts. Hum Gene Ther. 2010;21:1045-56.

2. Hass R, Kasper C, Böhm S, Jacobs R. Different populations and sources of human mesenchymal stem cells (MSC): a comparison of adult and neonatal tissue-derived MSC. Cell Commun Signal. 2011;9:12.

3. Griffin MD, Ryan AE, Alagesan S, Lohan P, Treacy O, Ritter T. Anti-donor immune responses elicited by allogeneic mesenchymal stem cells: what have we learned so far? Immunol Cell Biol. 2013;91:40-51.

4. Tchkonia T, Zhu Y, van Deursen J, Campisi J, Kirkland JL. Cellular senescence and the senescent secretory phenotype: therapeutic opportunities. J Clin Invest. 2013;123:966-72.

5. LeBrasseur NK, Tchkonia T, Kirkland JL. Cellular senescence and the biology of aging, disease, and frailty. Nestle Nutr Inst Workshop Ser. 2015;83:11-8.

6. Vekic J, Zeljkovic A, Stefanovic A, Jelic-Ivanovic Z, Spasojevic-Kalimanovska V. Obesity and dyslipidemia. Metabolism. 2019;92:71-81.

7. Kim SR, Eirin A, Zhang X, Lerman A, Lerman LO. Mitochondrial protection partly mitigates kidney cellular senescence in swine atherosclerotic renal artery stenosis. Cell Physiol Biochem. 2019;52:617-32.

8. Martindale JL, Holbrook NJ. Cellular response to oxidative stress: signaling for suicide and survival. J Cell Physiol. 2002;192:1-15.

9. Zhu X, Ma S, Eirin A, Woollard JR, Hickson LJ, Sun D, Lerman A, Lerman LO. Functional plasticity of adipose-derived stromal cells during development of obesity. Stem Cell Transl Med. 2016;5:893-900.

10. Eirin A, Riester SM, Zhu X, Tang H, Evans JM, O'Brien D, van Wijnen AJ, Lerman LO. MicroRNA and mRNA cargo of extracellular vesicles from porcine adipose tissue-derived mesenchymal stem cells. Gene. 2014;551: 55-64.

11. Eirin A, Zhu X, Puranik AS, Tang H, McGurren KA, van Wijnen AJ, Lerman A, Lerman LO. Mesenchymal stem cell-derived extracellular vesicles attenuate kidney inflammation. Kidney Int. 2017;92:114-24.

12. Meng $Y$, Eirin A, Zhu X, Tang H, Chanana P, Lerman A, Van Wijnen AJ, Lerman LO. The metabolic syndrome alters the miRNA signature of porcine adipose tissue-derived mesenchymal stem cells. Cytometry A. 2018;93:93103.

13. Lai RC, Chen TS, Lim SK. Mesenchymal stem cell exosome: a novel stem cell-based therapy for cardiovascular disease. Regen Med. 2011;6:481-92.

14. Bagno L, Hatzistergos KE, Balkan W, Hare JM. Mesenchymal stem cell-based therapy for cardiovascular disease: progress and challenges. Mol Ther. 2018; 26:1610-23.

15. Eirin A, Zhu X, Puranik AS, Woollard JR, Tang H, Dasari S, Lerman A, van Wijnen AJ, Lerman LO. Comparative proteomic analysis of extracellular vesicles isolated from porcine adipose tissue-derived mesenchymal stem/ stromal cells. Sci Rep-Uk. 2016;6:36120.

16. Nargesi AA, Lerman LO, Eirin A. Mesenchymal stem cell-derived extracellular vesicles for renal repair. Curr Gene Ther. 2017;17:29-42.

17. Zhang L, Zhu X, Zhao Y, Eirin A, Liu L, Ferguson CM, Tang H, Lerman A, Lerman LO. Selective intrarenal delivery of mesenchymal stem cell-derived extracellular vesicles attenuates myocardial injury in experimental metabolic renovascular disease. Basic Res Cardiol. 2020;115:16.

18. Zhu X, Chade AR, Rodriguez-Porcel M, Bentley MD, Ritman EL, Lerman A, Lerman LO. Cortical microvascular remodeling in the stenotic kidney: role of increased oxidative stress. Arterioscler Thromb Vasc Biol. 2004;24: $1854-9$.

19. Levey AS, Coresh J, Greene T, Stevens LA, Zhang YL, Hendriksen S, Kusek JW, Van Lente F, Chronic KDEC. Using standardized serum creatinine values in the modification of diet in renal disease study equation for estimating glomerular filtration rate. Ann Intern Med. 2006;145:247-54.

20. Sun Z, Evans J, Bhagwate A, Middha S, Bockol M, Yan H, Kocher J. CAPmiRSeq: a comprehensive analysis pipeline for microRNA sequencing data. BMC Genomics. 2014;15:423.

21. Saad A, Zhu X, Herrmann S, Hickson L, Tang H, Dietz AB, van Wijnen AJ, Lerman $L$, Textor $S$. Adipose-derived mesenchymal stem cells from patients with atherosclerotic renovascular disease have increased DNA damage and reduced angiogenesis that can be modified by hypoxia. Stem Cell Res Ther. 2016;7:128.

22. Tang Y, Zhang C, Liu H, Zhou Y, Wang Y, Li Y, Han Y, Wang C. Ginsenoside Rg1 inhibits cell proliferation and induces markers of cell senescence in CD34+CD38- leukemia stem cells derived from KG1a acute myeloid leukemia cells by activating the Sirtuin 1 (SIRT1)/tuberous sclerosis complex 2 (TSC2) signaling pathway. Med Sci Monit. 2020;26:e918207.

23. Lerman LO, Schwartz RS, Grande JP, Sheedy PF, Romero JC. Noninvasive evaluation of a novel swine model of renal artery stenosis. J Am Soc Nephrol. 1999;10:1455-65.

24. Meng Y, Eirin A, Zhu X, Tang H, Hickson LJ, Lerman A, van Wijnen AJ, Lerman LO. Micro-RNAS regulate metabolic syndrome-induced senescence in porcine adipose tissue-derived Mesenchymal stem cells through the P16/ MAPK pathway. Cell Transplant. 2018;27:1495-503.

25. Peng $S$, Gao D, Gao C, Wei P, Niu M, Shuai C. MicroRNAs regulate signaling pathways in osteogenic differentiation of mesenchymal stem cells (review). Mol Med Rep. 2016;14:623-9.

26. Baglio SR, Rooijers K, Koppers-Lalic D, Verweij FJ, Pérez Lanzón M, Zini N, Naaijkens B, Perut F, Niessen HWM, Baldini N, Pegtel DM. Human bone marrow- and adipose-mesenchymal stem cells secrete exosomes enriched in distinctive miRNA and tRNA species. Stem Cell Res Ther. 2015;6:127.

27. Borghesan M, Fafián-Labora J, Eleftheriadou $\mathrm{O}$, Carpintero-Fernández $\mathrm{P}$, Paez-Ribes M, Vizcay-Barrena G, Swisa A, Kolodkin-Gal D, Ximénez-Embún P, Lowe $R$, et al. Small extracellular vesicles are key regulators of non-cell autonomous intercellular communication in senescence via the interferon protein IFITM3. Cell Rep. 2019;27:3956-71.

28. Mulcahy LA, Pink RC, Carter DRF. Routes and mechanisms of extracellular vesicle uptake. J Extracell Vesicles. 2014;3:10-3402.

29. Markopoulos GS, Roupakia E, Tokamani M, Vartholomatos G, Tzavaras T, Hatziapostolou M, Fackelmayer FO, Sandaltzopoulos R, Polytarchou C, Kolettas E. Senescence-associated microRNAs target cell cycle regulatory genes in normal human lung fibroblasts. Exp Gerontol. 2017:96:110-22. 
30. Yang $Q$, Guo X, Cheng Y, Wang Y. MicroRNA-143-5p targeting eEF2 gene mediates intervertebral disc degeneration through the AMPK signaling pathway. Arthritis Res Ther. 2019;21:97.

31. Kim SR, Jiang K, Ogrodnik M, Chen X, Zhu X, Lohmeier H, Ahmed L, Tang $H_{\text {, }}$ Tchkonia T, Hickson $L$, et al. Increased renal cellular senescence in murine high-fat diet: effect of the senolytic drug quercetin. Transl Res. 2019;213: $112-23$.

32. Luo C, Zhou S, Zhou Z, Liu Y, Yang L, Liu J, Zhang Y, Li H, Liu Y, Hou FF, Zhou L. Wnt9a promotes renal fibrosis by accelerating cellular senescence in tubular epithelial cells. J Am Soc Nephrol. 2018;29:1238-56.

33. Bonab MM, Alimoghaddam K, Talebian F, Ghaffari SH, Ghavamzadeh A, Nikbin B. Aging of mesenchymal stem cell in vitro. BMC Cell Biol. 2006;7:14.

34. Johnsen KB, Gudbergsson JM, Andresen TL, Simonsen JB. What is the blood concentration of extracellular vesicles? Implications for the use of extracellular vesicles as blood-borne biomarkers of cancer. Biochim Biophys Acta Rev Cancer. 2019;1871:109-16.

\section{Publisher's Note}

Springer Nature remains neutral with regard to jurisdictional claims in published maps and institutional affiliations.

Ready to submit your research? Choose BMC and benefit from:

- fast, convenient online submission

- thorough peer review by experienced researchers in your field

- rapid publication on acceptance

- support for research data, including large and complex data types

- gold Open Access which fosters wider collaboration and increased citations

- maximum visibility for your research: over $100 \mathrm{M}$ website views per year

At BMC, research is always in progress.

Learn more biomedcentral.com/submissions 